\title{
El escenario del autoconsumo: una mirada femenina desde una comunidad rural de Oaxaca
}

\author{
María Eufemia Pérez Flores \\ mperezf@ipn.mx \\ Instituto Politécnico Nacional, \\ Centro Interdisciplinario de investigación \\ para el Desarrollo Integral Regional \\ unidad Oaxaca (CIIDIR-unidad Oaxaca. IPN)
}

\section{RESUMEN}

El modelo económico vigente en México ha provocado cambios en las estrategias de supervivencia de las comunidades rurales, los cuales se observan en los patrones de producción y consumo de dichos alimentos. El objetivo del artículo fue identificar la importancia nutritiva del consumo de alimentos denominados de "autoconsumo" en los hogares seleccionados; así como describir la percepción que tiene la población femenina con respecto a los factores sociales que contribuyen a la disminución de la producción y consumo de estos alimentos en una comunidad rural indígena de Oaxaca, México. Se trató de un estudio cuali-cuantitativo en el cual se aplicó una encuesta con una batería de preguntas de información socio-económica y sociodemográfica, una encuesta de recordatorio de 24 horas (EDR24hr) y una entrevista estructurada a una submuestra. Los principales resultados muestran que actualmente los alimentos de autoconsumo forman parte importante de la dieta de las familias estudiadas, se concluye que los factores que están contribuyendo a la pérdida de la producción de alimentos de autoconsumo en el municipio de estudio son los relacionados con el modelo neoliberal, ya que este sistema no considera a las poblaciones indígenas como sujetos de apoyo, si no como beneficiarios de políticas sociales.

Palabras clave: alimentación; percepción comunitaria; comunidad indígena. 


\title{
The scenario of self-consumption: a female perspective from a rural community in Oaxaca
}

\begin{abstract}
The current economic model in Mexico has caused changes in the survival strategies of rural communities, which are observed in the production and consumption patterns of said foods. The objective of the article was to identify the nutritional importance of the consumption of foods called "self-consumption" in the selected households; as well as to describe the perception that the female population has regarding the social factors that contribute to the decrease in the production and consumption of these foods in an indigenous rural community of Oaxaca, Mexico. It was a quali-quantitative study in which a survey was applied with a battery of questions of socio-economic and sociodemographic information, a 24-hour recall survey (EDR24hr) and a structured interview to a subsample. The main results show that currently self-consumption foods are an important part of the diet of the studied families, it is concluded that the factors that are contributing to the loss of self-consumption food production in the study municipality are those related to the model neoliberal, since this system does not consider indigenous populations as subjects of support, but rather as beneficiaries of social policies.
\end{abstract}

Keywords: feeding; community perception; indigenous community

Artículo recibido: 10 Setiembre. 2021 Aceptado para publicación: 15 Octubre. 2021

Correspondencia: mperezf@ipn.mx Conflictos de Interés: Ninguna que declarar 


\section{INTRODUCCIÓN}

La globalización y las políticas gubernamentales neoliberales han impactado de diversas formas en actividades de producción de alimentos, pues por un lado, la política económica agropecuaria excluye a los productores de bajos ingresos en la lógica de que no son competitivos en el mercado, relegando a los productores campesinos a los programas sociales de combate a la pobreza, en lugar de ser considerados sujetos adecuados de una estrategia gubernamental de desarrollo económico (Sesia, 2001;Fox and Haight, 2010; López y Sandoval, 2017). Esto ha provocado un abandono de las actividades del campo, que a su vez han propiciado complejos procesos de transformación en las estrategias de supervivencia de las unidades domésticas de las poblaciones rurales e indígenas de México, ya que como consecuencia los campesinos están migrando de manera temporal, periódica o definitiva hacia las ciudades mexicanas, hacia los campos agro-industriales del norte del país o a los Estados Unidos, buscando empleos generalmente informales y mal remunerados en la construcción, la agricultura, el trabajo doméstico o en otros servicios y enviando remesas a las familias que se quedan en el lugar de origen (Albo y Díaz, 2011; Pardo y Dávila, 2017). Sin duda, estos factores han contribuido a que la producción de alimentos deje de ser una práctica que mejora el abasto en el hogar de las familias pobres, haciéndolas más dependientes de los ingresos monetarios y orillando a las familias a la trasformación de los patrones de alimentos, reduciendo el consumo de cereales leguminosas y verduras (FAO, 2015; Du Plooy, Schönfeldt, y Hall, 2016) y en consecuencia, poniéndolas en mayor riesgo a la inseguridad alimentaria.

Según la FAO (2012), 1,192,029 (22\%) de las familias mexicanas destinan la totalidad de sus productos agropecuarios a cubrir las necesidades alimentarias de sus integrantes. La mayoría de estos hogares se encuentran localizados en seis estados del centro - sur: Estado de Méx., Oaxaca, Puebla, Veracruz, Guerrero y Chiapas, por orden de predominancia. La producción de alimentos para autoconsumo en el medio rural oaxaqueño comprende las actividades relacionadas con el cultivo de cereales, hortalizas, otros vegetales y árboles frutales; además de la cría de animales. Dicha actividad contribuye a garantizar la seguridad alimentaria de los hogares, porque abastece en base de una dieta familiar culturalmente aceptada.

Según la FAO (2006) existe seguridad alimentaria cuando "todas las personas tienen en todo momento acceso físico y económico a suficientes alimentos inocuos y nutritivos para 
satisfacer sus necesidades y sus preferencias, a fin de llevar una vida activa y sana” (p.2) e inseguridad alimentaria es la probabilidad de una disminución drástica del acceso a los alimentos o los niveles de consumo, debido a riesgos ambientales y sociales, o una reducida capacidad de respuesta (FAO, 2011). De acuerdo con los datos de la Encuesta Nacional de Salud y Nutrición (Ensanut Oaxaca, 2012), el estado de Oaxaca es uno de los estados de la República Mexicana con mayor inseguridad alimentaria en el país, pues la padece el $80.7 \%$ de los hogares encuestados, siendo los hogares rurales los más afectados por esta situación (84.9\%).

En los Valles Centrales de Oaxaca, la migración es un elemento fundamental de la elaboración de estrategias de reproducción de la unidad doméstica campesina (Sánchez, 1995; Reyes et al., 2004; Reyes y Gijón, 2007). A raíz de la migración principalmente, la unidad doméstica pierde integrantes sobre todo los varones, por lo que el despoblamiento rural está conformando un proceso lento hacia una mayor participación femenina en la estructura demográfica, la jefatura de hogares, el trabajo remunerado y hasta en la organización social-comunitaria. El aumento del consumo de alimentos industrializados ha sido posible por la falta de corresponsabilidad en el trabajo de las mujeres quienes dedican menos tiempo a las actividades para la alimentación y cuidados del hogar (Soler y Pérez, 2014). Según la ENSANUT 2018-2019, se encontraron porcentajes altos en el consumo de alimentos no recomendados (industrializados) entre los adultos encuestados clasificados en las localidades rurales, alcanzando el $87.8 \%$ con las bebidas no lácteas endulzadas, otros resultados cereales dulces $29 \%$, botanas, dulces y postres $24.4 \%$, bebidas lactas endulzadas $16.4 \%$, comidas rápidas y antojitos mexicanos $12.7 \%$ y carnes procesadas $5.3 \%$.

Según el Registro Agrario Nacional (RAN, 2016), 1.9 millones de mujeres actualmente son dueñas de la tierra, quienes viven en Oaxaca, Veracruz, Chiapas, Guerrero, México y puebla. Se calcula que en el área rural habitan cerca de 3.6 millos de mujeres indígenas, considerada como depositarias ancestrales de la seguridad alimentaria en la nación (FAO, 2019).

Vista la importancia de la producción de alimentos para autoconsumo para muchos hogares rurales mexicanos y el papel que juega la mujer en esta producción y consumo, El objetivo del artículo fue identificar la importancia nutritiva del consumo de alimentos denominados de "autoconsumo" en los hogares seleccionados; así como describir la 
percepción que tiene la población femenina con respecto a los factores sociales que contribuyen a la disminución de la producción y consumo de estos alimentos en una comunidad rural indígena de Oaxaca, México

\section{ESTRATEGIAS METODOLÓGICAS}

\section{Localidad de estudio}

El estudio se realizó en la cabecera municipal de San Juan Guelavía, municipio situado en los Valles Centrales de Oaxaca, México, a 39 kilómetros de la capital del estado. Según datos del Censo de Población y Vivienda 2010, Guelavía cuenta con una población total de 3,059 habitantes, de los cuales el $63.3 \%$ habla Zapoteco. En este municipio las oportunidades de desarrollo económico son limitadas. La agricultura se ha abandonado paulatinamente porque no es costeable; pese a ello, las tareas agrícolas siguen siendo la principal actividad de la población, aunque éstas son realizadas casi exclusivamente para el autoconsumo (Zafra y López Rocha, 2009). Los campesinos producen principalmente maíz, garbanzo, frijol, tomate rojo y fruta. Según el Servicio de Información Agroalimentaria y Pesquera de la SAGARPA (2019), la superficie sembrada total fue de 579.32 hectáreas, de las cuales 440 son de maíz de grano (94.6\% son de temporal), 120. 5 de agave, 13 de alfalfa, 5.5 de garbanzo de grano y solo 0.152 de tomate rojo.

\section{MATERIAL Y MÉTODOS}

\section{Componente cuantitativo}

Se llevó a cabo una investigación cuali-cuantitativa. A nivel cuantitativo, el procedimiento fue el siguiente: primero se calculó el tamaño de la muestra con la siguiente fórmula: $\mathrm{n}=\left(\mathrm{Z}^{2} * \sigma^{2}\right) / \mathrm{e}^{2} \mathrm{y}$ con un nivel de confianza del $95 \%$, quedando un tamaño de muestra de 30 hogares, seleccionados según muestreo aleatorio simple. Para seleccionar la muestra, primeramente, se obtuvo el mapa de la localidad, se utilizó el trazo urbano del INEGI, el cual fue sobrepuesto sobre la fotografía aérea de Google Earth del mismo municipio, permitiendo de esta manera ubicar y enumerar las viviendas existentes sobre el trazo urbano; esto debido principalmente a la falta de un censo en la localidad. Los hogares fueron seleccionados por sorteo (Hernández-Sampiere, et al.).

En cada uno de estos hogares, se encuestó a amas de casa de familia quienes debían cubrir con los siguientes criterios: haber habitado en la localidad de estudio por lo menos durante 10 años, ser adultas (mayores de 20 años y menores de 60 años), haber otorgado un consentimiento verbal de su participación voluntaria, y ser capaz física y mentalmente de 
responder las preguntas. la encuesta constaba de una batería de preguntas sociodemográficas como: edad, número de integrantes de la familia, escolaridad, lengua y ocupación; las preguntas socioeconómicas fueron sobre las diferentes fuentes de ingresos.

\section{Consumo de alimentos de autoconsumo}

Para conocer sobre el consumo de alimentos denominados de "autoconsumo" en la población de mujeres de la muestra, se aplicó una EDR24hr. La encuesta se procuró levantar de martes a sábado, debido a que éstos son días representativos del consumo habitual de una persona, además de que se evitó al máximo encuestar en los días posteriores a las festividades (Shamah, Villapando, y Rivera, 2006), adicionalmente se les preguntaba si el alimento consumido en la EDR24 era de autoconsumo. Se utilizaron las tablas del Sistema Mexicano de Equivalentes 4ta. edición (Pérez-Lizaur, PalaciosGonzález-Palacios, Castro-Becerra, y Flores-Galicia, 2014) y tablas de valor nutritivo de referencia nacional (INSP, 2012) para calcular el valor nutritivo de los alimentos de autoconsumo ingeridos, con respecto al consumo de energía, proteínas, carbohidratos, grasa, hierro, calcio y fibra.

\section{Captura de información y análisis}

Se creó una base de datos en el programa SPSS V.21. Se presenta un análisis descriptivo con los números, frecuencias y promedios de las variables mostradas en la tabla 1.

\section{Componente cualitativo}

De acuerdo con los ingresos totales que declaró cada hogar, se clasificaron tres niveles socioeconómicos (NSE) según cálculo en terciles: NSE alto, NSE medio y NSE bajo. Se seleccionó así una submuestra de tres hogares por cada NSE, quedando así un total de nueve hogares para ser entrevistados. Dentro de cada hogar se seleccionó a una participante que debía cubrir como principal requisito: ser adulta o adulta mayor, tener por lo menos 10 años de vivir en la localidad, haber dado consentimiento verbal de su participación voluntaria, así como haber permitido ser audio grabada, y haber respondido a la batería de preguntas sociodemográficas incluidas en las encuestas levantada durante la investigación cuantitativa.

Este componente utilizó la técnica de entrevistas estructuradas (Taylor, 1994) con adultos entre 20 y 88 años, habitantes de la localidad de estudios. 
Se elaboró un guion de entrevista centrándose en las experiencias y percepciones de la gente. Los temas fueron: productos cultivados en el pasado y actualmente, extensión de las tierras para los cultivos, participación familiar en la producción agropecuaria, costobeneficio de la producción de alimentos, consumo de alimentos producidos en el hogar, participación y opiniones acerca de los programas gubernamentales, fuentes de empleo, disponibilidad y consumo de alimentos, y cambios en los patrones de consumo.

Es importante mencionar que la dimensión temporal abarcada en las entrevistas, en cuanto a procesos de cambios entre un "antes" y el "ahora”, aun si se manejó oficialmente en las preguntas como una diferencia de una década, en el imaginario de las personas que respondieron fue visualizado en casi todos los casos con una temporalidad mayor de quince, veinte o hasta treinta y cinco años atrás, pero siempre siendo significativa para sus propias vidas, como eventos presenciados por los y las entrevistadas.

Previa autorización de los participantes, todas las entrevistas fueron audio grabadas y después transcritas en archivos electrónicos a partir de los cuales se generaron categorías de información mediante codificación abierta. Posteriormente, se llevó a cabo un análisis de las categorías centrales y finalmente las categorías fueron ubicadas junto con los contenidos correspondientes en matrices temáticas.

\section{RESULTADOS Y DISCUSIÓN}

\section{Componente cuantitativo}

La información obtenida durante la encuesta arrojó los siguientes resultados: de un total de 127 integrantes de los 30 hogares incluidos en el estudio, 64 (50.4\%) fueron varones y $63(49.6 \%)$ mujeres. Los hogares estaban formados por 3.9 integrantes promedio.

Con respecto a la edad promedio en los hogares, las estadísticas descriptivas muestran que la edad de las madres de familia (50.4 años) es ligeramente menor que la de los padres (52.3 años). El nivel de escolaridad promedio de los integrantes mayores de 15 años fue de 6.7 años, siendo menor para las mujeres (6.5 años) que para los hombres (6.9 años). Los encuestados mencionaron las siguientes ocupaciones: trabajo en el campo, 20.6\%; ama de casa, $11.8 \%$; empleado(a), $11.8 \%$; estudiantes, $6.8 \%$; comerciantes, $22.5 \%$; el $19.6 \%$ empleados en servicios o en oficios; artesanos(as), 2.9\%; y los que no tuvieron ninguna actividad $3.9 \%$.

El ingreso promedio anual de los hogares fue de 80,986 pesos mexicanos equivalente a 2.15 salarios mínimos vigentes, estos ingresos provienen de la combinación de diferentes 
estrategias de supervivencia a nivel de núcleo doméstico: sueldos por trabajo asalariado ya sea dentro o fuera de la localidad (59.5\%), ingresos por actividades comerciales $(23.9 \%)$, transferencias monetarias por programas sociales gubernamentales $(14.9 \%) \mathrm{e}$ ingresos por remesas migratorias en especie y en efectivo (1.7\%).

Por otra parte, a los adultos y adultos mayores de los hogares se les aplicó una encuesta para conocer sobre la ingesta de alimentos denominados de "autoconsumo", el total de la muestra fue de 60 a quienes se les aplicó una EDR24 hrs., dicha población fue conformada con $41.7 \%(n=25)$ de mujeres y $58.3 \%(n=35)$ de hombres. Los resultados de la encuesta mostraron que el $40 \%$ de las personas encuestadas consumieron algún alimento de autoconsumo. Se encontró que la mayoría acostumbra a comer preparaciones a base de maíz y sus derivados como son tortillas, atole, tamales, memelas, téjate y como espesante de guisados. Los productos de origen animal que se aprovechan son: queso, leche y chapulines. Otros productos vegetales que se aprovechan son los nopales, calabazas y chepiles. En la figura 1 se muestra la frecuencia del consumo de estos alimentos en diferentes presentaciones entre los adultos del estudio.

Figura 1. Prevalencia (\%) de preparaciones a base de alimentos de autoconsumo en la población de estudio.

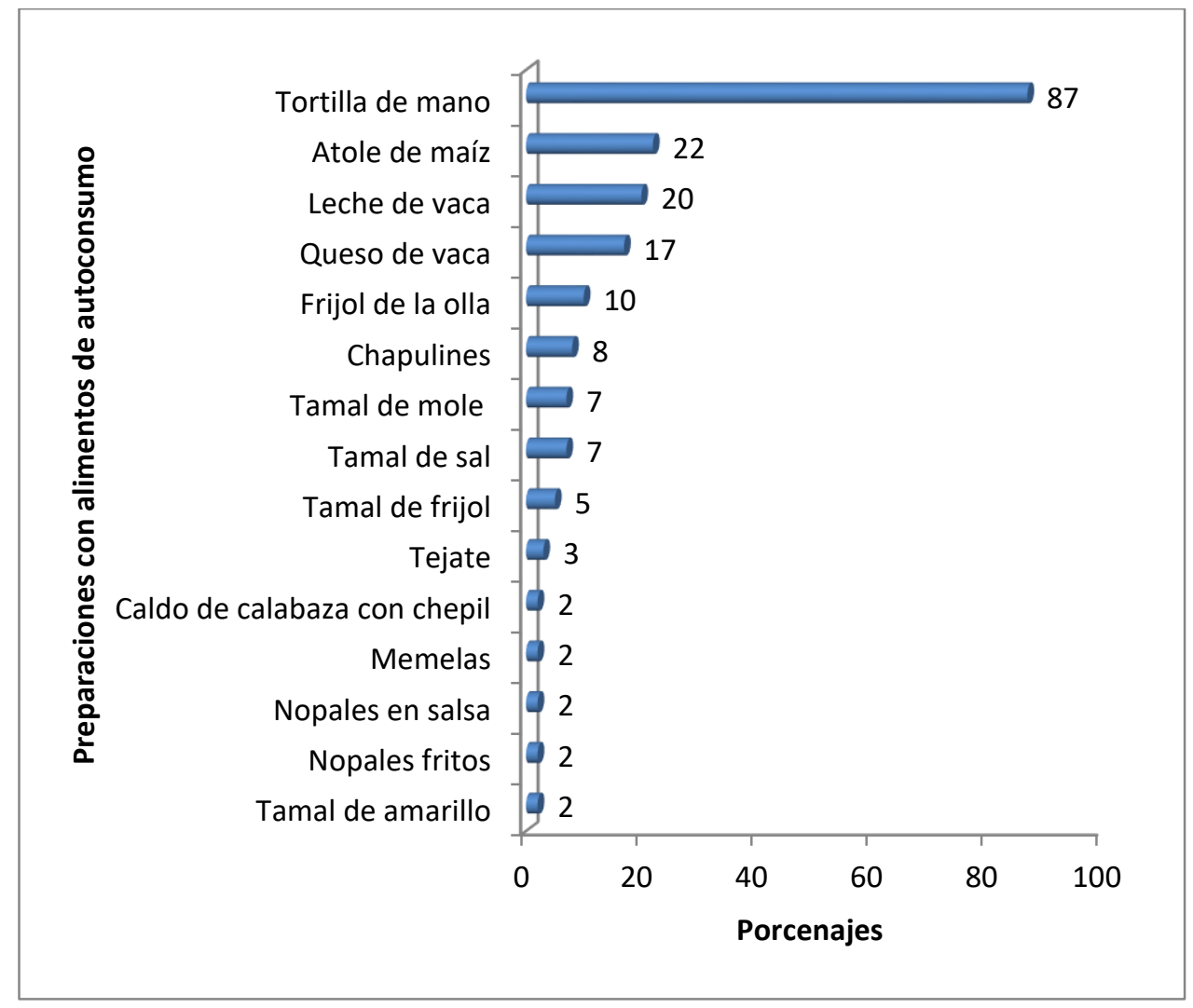


A pesar de que se suspendió la aplicación de la encuesta EDR24h por las festividades del día de muertos. Es posible que los resultados hayan sido sesgados ya que se observó que las preparaciones de alimentos que forman parte de este rito se realizaban previamente a los días establecidos como de festejo. Habría que recordar al respecto que, contrariamente de las profundas trasformaciones que han impactado el medio rural mexicano, se mantienen las tradiciones, especialmente las alimenticias, entre las cuales el maíz sigue proporcionando la base fundamental para platillos regionales y estacionales que se consumen especialmente en las fiestas patronales religiosas (Kato et al., 2009).

Este resultado se asocia a la presencia y disponibilidad de estos alimentos en el contexto productivo local; un resultado en línea con lo que establecen Furst et. al. (1996), al considerar el factor de la producción y disponibilidad local como uno de los que más influyen en la decisión del consumo de los alimentos.

Por otro lado, se calculó el valor nutritivo de la dieta de los adultos, encontrándose un consumo energético total medio de $1651 \mathrm{kcal} /$ día y un consumo energético medio de los alimentos de "autoconsumo" de 925kcal/día (mínimo de 232Kcal/día y un máximo de $3362 \mathrm{kcal} /$ día), lo que representa un aporte significativo del 56\% de las Kcal/día y el $46 \%$ de las calorías promedio requeridas según el grupo de edad.

\section{Características generales de las entrevistadas}

En lo que concierne a las mujeres a quienes se les aplicó la entrevista cualitativa, se encontró que tenían una media de edad de 48 años y de escolaridad de 6.7 años. La religión que todas las entrevistadas practican es la católica. Siete de las entrevistadas mencionaron ser bilingües zapoteco/español, mientras que una de las mujeres sólo habla el español. El $77.7 \%$ de las entrevistadas reportaron ser casadas, $11.1 \%$ solteras y $11.1 \%$ viudas.

Las ocupaciones principales de las mujeres entrevistadas fueron: como empleadas fuera de la localidad y amas de casa $22.2 \%$, elaboración de tortillas y ama de casa $11.1 \%$, tienen un negocio propio como pollería y tlapalería y además son amas de casa $22.2 \%$, actividades de agricultura y amas de casa $33.3 \%$.

\section{Causas de la disminución de la producción de alimentos}

\section{1) Productos cultivados antes y ahora}

Con respecto a la producción de alimentos en el campo, seis de las nueve mujeres entrevistadas opinaron que hasta el momento de la entrevista seguían cultivando lo mismo 
que "antes". Las otras tres personas dijeron que antes se cultivaban más productos alimenticios que ahora y que ya no se cultiva lo mismo por las siguientes razones: los campesinos han optado por abandonar la actividad agrícola por diferentes oficios, sobre todo el de albañilería, la presencia de la lluvia ha disminuido y los jóvenes de hoy ya no quieren trabajar en el campo.

\section{2) Propiedad de la tierra disponible para siembra}

Con respecto a la disponibilidad de tierra para cultivos, cinco mujeres afirmaron que ha disminuido su extensión, en comparación con las extensiones que tenían disponibles hace 10 años. Los porqués de la disminución de la tierra disponible para siembra incluyen las siguientes razones: en un caso se tuvo que vender para cubrir gastos de una fiesta, en dos casos se tuvo que vender para cubrir necesidades básicas, en otro caso se perdió el terreno por la muerte del padre sin testamento y otra más de las entrevistadas reportó que ha disminuido su extensión de tierra porque fue repartida entre familiares. Las entrevistadas hacen referencia a la disminución del acceso de la tierra, factor que afecta a la autosuficiencia, el acceso a los alimentos, las oportunidades económicas de los hogares, la seguridad alimentaria y los niveles de pobreza (Ramírez, Prada y Useche, 2000; Lacy, 2002).

\section{3) Participación familiar en la producción de alimentos}

Todas las entrevistadas dijeron que hace 10 años tenían algún familiar que se encargaba del trabajo en el campo y nadie usaba apoyo de otras personas ajenas a la familia. Las respuestas que dieron a la pregunta ¿quiénes trabajan en el campo ahora? muestran que la actividad familiar en el campo está claramente disminuyendo. Dos de las personas entrevistadas dijeron no tener ningún integrante de la familia que trabaje en el campo debido a que ya no se realiza esta actividad en su hogar. De entre las personas entrevistadas que mencionaron que sí había alguien en el hogar que trabajara en el campo, cinco dijeron que se trataba de algún familiar como el padre, el esposo, los hijos o el tío, pero dos mujeres señalaron utilizar un "mediario" para el trabajo del campo; esto, por no contar con el apoyo del marido como lo expresa una de ellas:

"Hoy en día, ahora sí que lo trabaja el "mediario" que se llama, tenemos una persona que se le llama aquí el "mediario", él trabaja el terreno que posee hoy en día uno el que se encarga de hacer todo ese trabajo. Este... mediario significa pues a la vez yo pongo una parte 
y él otra parte, en ambas partes estamos asumiendo el trabajo del campo, ajá, o sea, que ahí formamos dos partes tanto la dueña como el “mediario" ........Porque soy mujer, es por esa razón, entonces pasa eso porque yo tengo una posición de mujer y para no tener los terrenos abandonados pues hay que procurar de que la gente lo haga y mientras tanto nosotros como dueñas estamos al tanto." [mujer de 42 años].

Las principales razones que dan las entrevistadas y el entrevistado frente a los cambios en el trabajo del campo se atribuyen al fallecimiento de padres y esposos y la falta de algún familiar interesado en sustituir en el trabajo. En uno de los casos la partida de los hijos cuando se casaron representó el abandono de la producción del campo por parte del hogar.

Según Garine (1995), "los hábitos alimentarios se definen como el estudio de los medios por los que los individuos responden a presiones sociales y culturales, seleccionan, consumen y utilizan porciones del conjunto de alimentos disponibles". Se ha señalado que, a medida que los grupos locales abandonan la agricultura para autoconsumo, dependen de manera creciente de la compra de alimentos (Martínez, 1993) y que, para hacer frente al encarecimiento de los alimentos, suelen reducir los gastos en salud y educación, además de cambiar sus patrones de alimentación pues consumen alimentos de menor precio, principalmente cereales como pan y tortilla. Es así como se abandona o se disminuye el consumo de alimentos ricos en micronutrientes tales como la leche, la carne o las frutas y hortalizas (FAO, 2012). De acuerdo con esta premisa, si bien es cierto que la producción de alimentos para autoconsumo no logra responder por completo a las necesidades de consumo de alimentos de los hogares, esta práctica forma parte de un conjunto de respuestas tendientes a superar las privaciones de los hogares, pues asegura de forma parcial el consumo de energía y micronutrientes a las familias.

\section{4) Costo alto y bajas ganancias en la producción de los alimentos}

Una de las mujeres comentó además acerca de la problemática de la falta de ganancias en la venta de los productos del campo, ya que producirlos cuesta más de lo que se pueda obtener en la venta, o más de lo que cuesta comprar los alimentos primarios en las tiendas de Diconsa, como es el caso del maíz. Esto no es más que un reflejo del conjunto de políticas públicas que conllevan a la reducción del gasto público en la agricultura en el país (FAO, 2012) y al abandono del campo. 
También se mencionó por dos entrevistadas que se paga muy poco el jornal de trabajo en el pueblo. Otro problema de costo-beneficio mencionado fue la queja de que el trabajo en el campo es muy arduo y que las ganancias derivadas del campo no dan suficiente para vivir.

\section{5) Patrones de producción de alimentos}

Los adultos entrevistados mencionaron que "antes", producían los siguientes alimentos: maíz, frijol blanco, frijol rojo, frijol negro, garbanzo, calabacitas y semilla de calabaza; como productos del traspatio contaban con cerdos, gallinas, guajolotes, huevos, rábanos y lechugas. Se nota que la producción de autoconsumo proveniente del campo ha disminuido, ya que los productos de autoconsumo que actualmente reportan son sólo: maíz, frijol, calabacitas y semilla de calabaza, mientras que los cultivos como el garbanzo y los otros tipos de frijol se perdieron. Pero tal parece que no sucede lo mismo para los productos de traspatio, porque las entrevistadas mencionaron tener más productos actualmente de los que se tenían hace diez años, incluyendo a cerdos, gallinas, guajolotes, borregos, patos, huevos, acelgas, perejil, hierba santa, papaya, epazote, hierba buena, chiles, rábanos y tomates.

Las entrevistadas y el entrevistado creen que los cambios en la producción de autoconsumo han sido propiciados por el hecho de que la gente ha dejado de comer estos productos y por lo tanto han prescindido de ellos en la alimentación básica. También mencionaron como una causa muy importante de las transformaciones en la producción agrícola, la falta de interés entre los jóvenes para trabajar el campo y sustituir a los adultos cuando ellos se vuelven mayores o fallecen. Por otro lado, el aumento en la variedad de los alimentos de autoconsumo del traspatio se debe quizás a lo que señala una señora:

“...Antes la gente no sabía cultivar las cosas porque se compraban y ahora ya nos enseñaron a tener huertos familiares, eso es lo que nos enseñaron en la clínica...Ha cambiado la producción en la casa pues, en las verduras, porque nos enseñaron a meter rábanos en la casa, calabacita..." [mujer de 48 años].

\section{6) Ventajas y desventajas de los programas de gobierno}

En cuanto al uso que se les da actualmente a las trasferencias monetarias del Programa Oportunidades o Prospera, una de las entrevistadas opinó que dichos recursos son utilizados inadecuadamente por los beneficiarios: 
"Los padres de familia no usan los recursos de Oportunidades o Prospera, lo usan para otras cosas que no van....... no lo usan en la cuestión que debe de ser para lo que tiene que ser que el presidente lo manifestó en la televisión..." [mujer de 42 años].

Son tres las entrevistadas que opinaron que los beneficios adquiridos por Oportunidades o Prospera sirven para solventar sus necesidades:

“...Es un programa que sirve mucho para proveer las necesidades de mi hija"'[mujer de 42 años].

“...Es un recurso que nos da el gobierno, que no es porque nos lo regala que es el mismo sueldo que le quitan a los trabajadores nos los dan... pero cuando lo recibimos compramos lo que necesitamos..." [mujer de 57 años].

“...Es mejor que sigan apoyando porque sí, nos ayuda” [mujer de 38 años].

A la pregunta si Oportunidades o Prospera ayuda con la alimentación de los hogares que reciben el programa, cuatro contestaron rotundamente que sí ayuda. Los porqués de esta respuesta se anotan a continuación: porque es dinero que no llega a sus hogares por otras fuentes; porque cuando llega sirve para comprar cosas para comer; y porque apoya a mujeres que lo necesitan. Así lo expresó una de las mujeres:

“...Porque a veces en la actualidad pues no hay trabajo, por ejemplo, las mujeres hoy en día trabajan, hay mujeres a veces que no tienen trabajo y tienen dos niños o tres niños o tienen trabajo, así como intercalando los días, entonces ¿qué pasa? ese apoyito que les da Oportunidades pues ya solventa esos días que ellas no tienen trabajo, de esa manera utilizan el dinero también y es un apoyo muy enorme que les solventa esos días que ellas no trabajan... " [mujer de 42 años].

Tres entrevistadas expresan que Oportunidades o Prospera sí ayuda a la alimentación, mientras se le dé un buen uso a ese recurso. Según ellas, hay mujeres que no saben distribuir el apoyo en el hogar y existen estudios que apoyan estas percepción, donde se halló que el programa aumenta la adquisición de alimentos ricos en energía, atribuibles al aumento de ingresos generados por las trasferencias monetarias pero que las pláticas del programa sí tienen efecto en la identificación de la dieta apropiada y la obtención de 
energía procedente de productos animales y de frutas y verduras (Hoddinot y Skoufias, 2000 y Villatoro, 2005). Contrario a esto, en una investigación más reciente se halló que existe una asociación positiva entre las transferencias monetarias altas de beneficiarios del programa con un mayor índice de masa corporal (IMC) y presencia de obesidad y sobrepeso en la población adulta (Fernal, Gertler, y Hou, 2008).

La percepción de dos entrevistados fue que Oportunidades o Prospera no ayuda a la alimentación, ya que opinan que la gente sigue igual y no usa este apoyo para los alimentos, sino para comprar ropa o para invertir en negocios, no obstante, el impacto de dicho programa en la disminución de la pobreza no ha sido efectivo (Rodríguez y Patrón, 2017).

Es de considerar la opinión muy particular del único hombre entrevistado que culpa al programa por el abandono del trabajo:

“...ayuda en un $25 \%$ porque lo demás lo emplean para otra cosa porque hay unos que dicen - el gobierno ya mandó dinero y ya no [hay] trabajo..." [hombre 45 años].

Es interesante notar que sea el único varón entrevistado en opinar lo anterior, sobre todo considerando que los apoyos de Oportunidades o Prospera llegan a las mujeres titulares y no a los varones, por lo que parece una opinión claramente marcada por la diferencia de género. Estas últimas percepciones son semejantes a las encontradas en el informe de evaluación del impacto social del programa (Adato, 2000).

\section{7) Nuevas oportunidades de trabajo}

Según la opinión de los entrevistados, en Guelavía han surgido cambios en los patrones laborales de la comunidad, los cuales corresponden al proceso de desagrarización (Escalante, 2008). Las respuestas de los entrevistados fueron que ha cambiado el trabajo en comparación con los que había “antes", siendo ésta una opinión generalizada del total de los entrevistados. Estos se lo atribuyen a diversas situaciones, entre ellas: los cambios de actividades entre los hombres, ya que hoy en día es más común que se dediquen a la construcción que al campo; la presencia de transporte constante que permite salir a Tlacolula o a la ciudad de Oaxaca a trabajar; la posibilidad de trabajar en la localidad en la construcción de casas; la "falta" de trabajo en el campo; y la presencia de lo que se identifica como "pereza" y/o negación al trabajo manual duro como el agrícola, sobre todo entre los más jóvenes. 
Al preguntar si hay un mayor acceso a nuevos trabajos en la actualidad, la mayoría opinó que sí, mencionando que los nuevos trabajos disponibles son de albañiles, peones de albañiles y herreros entre los varones. Para las mujeres, se mencionó el empleo para limpieza de casas y como empleadas de mostrador en tiendas. Las opciones mayores de trabajo y generación de ingresos se puede decir que ayuda al incremento de la variedad de los alimentos, pero no a la producción de los alimentos (Uribe, et. al., 2007). Lo anterior refiere la existencia de unidades económica familiar pluriactivas (Cartón de Grammont, 2009).

Con respecto a la relación entre la alimentación y los ingresos por los nuevos trabajos, en su mayoría los entrevistados mencionaron que los nuevos trabajos contribuyen a mejorar la alimentación en el hogar, opinando que, a pesar de que les pagan poco dinero, sí hay recursos para comer, y que se supone que los trabajos de hoy en día son para mejorar las condiciones del hogar incluyendo la alimentación.

\section{8) Mayor disponibilidad de alimentos}

Cuentan los entrevistados que eran pocas las tiendas que existían antaño en la localidad, mencionando un número variable de entre dos y seis. Según ellos, en esas tiendas se vendían productos alimenticios que se producían en el lugar como maíz, frijol y queso de producción local y otros que se traían de fuera como el trigo, arroz, aceite, leche, cacao, azúcar, café, chocolate, sopa de pasta, harinas, sardina enlatada y chiles en vinagre; dos de las mujeres entrevistadas comentaron que se vendían también bebidas alcohólicas como el mezcal.

Cuando se les preguntó si creían que ha cambiado algo o si se sigue vendiendo lo mismo en las tiendas, todos los entrevistados contestaron que sí ha habido cambios en los productos a la venta. Ahora se venden más alimentos industrializados como jamón, chorizo, salchicha, tocino, salami, crema, queso y quesillo empaquetados, mantequilla, refrescos, frituras denominadas "chatarra", cereal de caja, sopa Maruchan, pan de caja, cerveza, mezcal, verduras, frutas, carnes y helados. Todos comentaron que estos productos provienen de la ciudad y/o de otros estados. Dos de ellos mencionaron explícitamente que ahora hay más tiendas que antes; una mujer dijo que ahora hay hasta dos tiendas por cuadra y otra más comentó que son como 40 o 50 las tiendas en todo el pueblo. 
Dos mujeres compartieron sus razones del porqué de los cambios en la oferta de alimentos en las tiendas:

“...Porque la gente sale más. Es más práctico hacer una sardina por eso la gente pide más, refrescos, enlatados y todo eso...” [mujer de 20 años].

“......que la comunidad [se] ha ampliado más ya hay más personas, ya hay más familias, ya aumentó más también. Es por esa razón entonces... aumentó más la gente y ya come más esos productos fríos..." [mujer de 42 años].

Como se puede apreciar, las razones aducidas tienen que ver con el desplazamiento cotidiano relacionado con el trabajo y los ritmos más rápidos en la vida diaria (Popkin, 2001) y a la creciente urbanización (FAO, 2012).

\section{9) Cambios en los patrones de consumo de alimentos}

Autores como Mintz (2003), consideran que las preferencias alimentarias pueden ser resistentes al cambio y Camarena, Saldoval y Domínguez (2011), apoyan esta idea afirmando que el consumidor de alimentos por instinto natural manifiesta rechazo a lo desconocido. Por otro lado, en estudios previos se han demostrados cambios en la dieta de los mexicanos, aumentando el consumo de alimentos industrializados propiciado por la urbanización y el efecto de los programas sociales (Rivera, Perichart y Moreno, 2012; García y Bermúdez, 2017; Olvera, Schmook, Radel y Nazar, 2017). En este estudio, la mayoría de los entrevistados (siete) cree que lo que se come actualmente es diferente a lo que se comía "antes". Los porqués incluyen las siguientes respuestas: ahora ya no se come natural; el precio de los alimentos es más alto; hay mayor variedad de alimentos; los alimentos de hoy contienen más grasas; y se considera también que son alimentos "rebajados", sobre todo en el caso de la leche.

Las razones aducidas del porqué han cambiado la dieta incluyen: "ahora hay dinero para comprar"; "hubo cambios en el clima"; "hay contaminación", y "hay mayor disponibilidad de los alimentos". Es de resaltar que dos de las mujeres entrevistadas dicen que no han cambiado su forma de comer y que siguen cocinando igual.

Dos de las entrevistadas comentaron que los jóvenes no comen diferente a los adultos de la localidad. El resto, sin embargo, opinaron que los jóvenes sí comen diferente a los adultos y que la diferencia consiste en que comen más productos industrializados y 
comidas rápidas en lugar de los guisos característicos del pueblo, a base del maíz y el frijol:

“...Ahora les gusta pura carne y golosina...” [mujer de 38 años].

“...Antes comíamos comidas sencillas, [ahora] los jóvenes exigen

pizza......" [mujer "2"de 42 años].

“...Consumen mucho refresco y muchas golosinas...” [mujer de 42 años].

Tres de las mujeres que sí creen que la comida entre jóvenes y adultos es diferente, comentaron que esto sucede desde que los jóvenes salieron al norte como migrantes. Otra más comentó que se trata de un fenómeno reciente y el señor comentó que el fenómeno se da desde que los padres lo permiten. Sólo dos de las entrevistadas trataron de responder al por qué de la diferente forma de comer y las respuestas fueron:

“...por la publicidad de tantos productos, ellos quieren imitar lo que ven en la TV y porque se quedan deslumbrados cuando conocen algo nuevo" [mujer de 42 años].

\section{CONCLUSIÓN O CONSIDERACIONES FINALES}

Los resultados del estudio muestran que el consumo de alimentos denominados de autoconsumo forma parte importante del aporte de nutrimentos en la dieta de los adultos del estudio.

Desde el punto de vista académico el presente estudio ha pretendido contribuir al aporte de información con respecto al tema del abandono de la producción de alimentos de autoconsumo, derivado de las impresiones de las personas entrevistadas, se concluye que los factores que están contribuyendo a la perdida de la producción de alimentos de autoconsumo en el municipio de San Juan Guardavía son los relacionados al modelo neoliberal, ya que no considera a las poblaciones indígenas como sujetos de apoyo, si no como beneficiarios de políticas sociales; esto se traduce en un ámbito en el que este tipo de poblaciones se generan procesos contradictorios y obstaculizadores en la producción de alimentos y exposición al cambio en los hábitos de consumo de alimentos y las relaciones sociales que contribuían a un sistema de alimentario de intercambio de alimentos y saberes con respecto a la producción, preparación y consumo de los alimentos. 
Por otro lado, podemos concluir que este estudio evidencia la complejidad de los aspectos que influyen en la alimentación de las poblaciones indígenas de Oaxaca. Sin embargo, deberán realizarse más investigaciones que permitan visualizar la relación de los factores socioeconómicos actuales provocados por el sistema neoliberal presentes en este tipo de comunidad con los patrones de alimentación.

Las corrientes agroecológicas y ecofeministas deben ser recurridas para contrarrestar los sesgos del sistema occidental, que generado una desarticulación de los sistemas alimentarios de los campesino y campesinas.

\section{LISTA DE REFERENCIAS}

Adato, M. (2000), El impacto de Progresa sobre las relaciones sociales en la comunidad, Washington, D.C., Instituto Internacional de Investigaciones sobre Políticas Alimentarias (IFPRI) recuperado de http://www.ifpri.org/sites/default/files/ publications/adato_comunidad.pdf.

Albo, A., y Díaz, J. (2011). La Migración Mexicana hacia los Estados Unidos: Una breve radiografía. Documentos de Trabajo. Recuperado de https://agenciabk.net/emigracion_mexicana.pdf

Beltran B.M. (2005). Cambio alimentario e identidad de los indígenas mexicanos (No. 8). UNAM. $123 \mathrm{pp}$.

Budhram, D., Gibson, R., Hilb, M., Birbaumer, G., Boullón, R., Gault, F., \& Axson, D. A. J. (2014). Panorama de la Seguridad Alimentaria y Nutricional en América Latina y el Caribe (No. E10-1520). FAO, San José (Costa Rica).

Camarena D., Sandoval S. \& Domínguez E. (2011) Actitud hacia el consumo de comidas étnicas/internacionales y tradicionales en el norte de México. Revista Agroalimen-taria, Vol.17, Num. 32, pp...87.

Escalante, R., Catalán, H., Galindo, L. M., y Reyes, O. (2011). Desagrarización en México: tendencias actuales y retos hacia el futuro. Escrito por. Cuadernos de Desarrollo Rural, 4(59), 87-116.

Fao. (2012). Boletín de Agricultura Familiar. Boletín de Agricultura Familiar, (Septiembre), 12. Retrieved from http://www.rlc.fao.org/uploads/media/baf_201209.pdf

Fernald, L. C., Gertler, P. J., \& Hou, X. (2008). Cash component of conditional cash transfer program is associated with higher body mass index and blood pressure in adults. The Journal of nutrition, 138(11), 2250-2257. 
Fox J, Haight L, (2010). La política agrícola mexicana: metas múltiples e intereses en conflicto. Fox J, y Haight L, (coords.) Subsidios para la desigualdad. Las políticas públi-cas del maíz en México a partir del libre comercio. Santa Cruz y Distrito Federal: CIDE y Woodrow Wilson International Center for Scholars.

Furst, T., Connors, M., Bisogni, C., Sobal, J. \& Winter Falk, L., 1996. Food choice: a conceptual model of the process. Appetite, Num. 26, pp. 247-266.

García, M. E., \& Bermúdez, G. (2017). La neocolonización del paladar en las décadas recientes\| Palate's Neocolonialism in Recent Decades. Razón y Palabra, 20(3_94), 106-118.

Garine, I. "Los aspectos socioculturales de la nutrición” en Contreras, J. (coord.) Alimentación y cultura: necesidades gustos y costumbres. Universidad de Barcelona, Barcelona, España 1995.

Gutiérrez JP, Rivera-Dommarco J, Shamah-Levy T, Villalpando-Hernández S, Franco A. y Cuevas-Nasu L. Encuesta Nacional de Salud y Nutrición 2012. Resultados Nacio-nales. Cuernavaca, México: Instituto Nacional de Salud Pública, 2012.

Hoddinott, J., Skouflas, E. y Washburn, R. (2000). El impacto de Progresa sobre el consumo: Informe final. En: Evaluación de resultados del programa educación, salud y alimentación. Secretaría de Desarrollo Social: México, D.F.

INEGI, X. (2010). Censo General de Población y Vivienda, México.

Instituto Nacional de Salud Pública. Bases de datos del valor nutritivo de los alimentos. Cuernavaca, México: INSP, 2012.

Kato, T. A., Mapes, C., Mera, L. M., Serratos, J. A., \& Bye, R. A. (2009). Origen y diversifi-cación del maíz: Una revisión analítica Comisión Nacional para el Conocimiento y Uso de la Biodiversidad (CONABIO)/UNAM: México, D.F.

Martínez, J. M. (1993). Consumo rural/consumo urbano ¿dónde están las diferencias?. Distribución y consumo, (8), 10-19.

Mintz, Sidney (2003) sabor a comida, sabor a libertad. Incursiones en la comida, la cultura y el pasado (PP. 1-173). CIESAS; CONACULTA y Ediciones de la Reina Roja.

Organización de las Naciones Unidas para la Agricultura y la Alimentación. (FAO) (2006), "Food security", FAO's Agriculture and Development Economics Division (ESA), (Policy Brief, 2). 
Organización de las Naciones Unidas para la Alimentación y la Agricultura (FAO). Panorama de la Seguridad Alimentaria y Nutricional 2012 en América Latina y el Caribe. Roma: FAO, 2012

Pérez-Lizaur, A. B., González-Palacios, B., Castro-Becerra, A. Flores-Galida (2015). Sistema mexicano de alimentos equivalentes $4^{\circ}$ edición. México.

Popkin, B. M. (2001). Symposium: Obesity in Developing Countries : Biological and Ecological Factors The Nutrition Transition and Obesity in the Developing World (1), 871-873.

Ramírez, Juan; PRADA, Sergio; Useche, Pilar. (2000). «Una descripción de la economía campesina en Colombia: las encuestas de calidad de vida y eficiencia 1997 1999». En: Coyuntura Colombiana, (67), 22-50.

Rivera J. A., Perichart O. y Moreno J. (2012). Determinantes de la obesidad, marco conceptual y evidencias científicas. En Rivera, J.A., Hernández, M., Aguilar, C., Vadillo, F., Murayama, C., (Ed.), Obesidad en México, recomendaciones para una política de esta-do. México, D.F. Universidad Autónoma de México. pp. 45-77

SAGARPA (2014). Servicio de Información Agroalimentaria y Pesquera. http://www.siap.gob.mx/

Shamah, L. T., Villapando, H. S., y Rivera, D. J. (2006). Manual de procedimientos para proyectos de nutrición.

Taylor, S.J. R. Bogdan. (1994). La observación participante: preparación del trabajo de campo. En Introducción a los métodos participativos de Investigación. Tercera edición. Buenos Aires, Ed. Paidos.

Uribe, Á., Mancilla López, M. C., Cortés Torres, L. P., y Elena, J. (2007). Caracterización Socioeconómica y Seguridad Alimentaria de los Hogares Productores de Alimentos para el Autoconsumo Antioquia-Colombia. Agroalimentaria, (25), $109-122$.

Villatoro, P. (2005). Programas de transferencias monetarias condicionadas: Experiencias en América Latina. Revista de la CEPAL, (86), 87-102.

Zafra, G. y López Rocha, M. (2009) Impacto de la migración en la juventud: San Juan Guelavía. Agricultura, Agricultura Sociedad y Desarrollo (6)1, 129-144. 
Sesia P. (2001). “Aquí la progresa está muy dura”: Estado, negociación e identidad entre familias indígenas rurales. Desacatos. Revista de Antropología Social. No. 8, pp. 109-128.

Carton de Grammont, H. (2009). La desagrarización del campo mexicano. Convergencia, 16(50), 13-55.

Olvera, B., Schmook, B., Radel, C., \& Nazar Beutelspacher, D. A. (2017). Efectos adversos de los programas de apoyo alimentario en los hogares rurales de Calakmul, Campeche. Estudios sociales (Hermosillo, Son.), 27(49), 11-46.

Du Plooy, Z., Schönfeldt, H. C., \& Hall, N. (2016). The role of traditional foods in foodbased dietary guidelines - A South African case study on maas (cultured milk). Food Chemistry, 238(2018), 22-28. https://doi.org/10.1016/j.foodchem.2017.04.044

Salazar, R. L., \& Godoy, S. A. S. (2018). La seguridad alimentaria en México: el reto inconcluso de reducir la pobreza y el hambre. Espacio abierto: cuaderno venezolano de sociología, 27(1), 125-148.

FAO. 2015. The State of Agricultural Commodity Markets 2015-16. Roma

Siliprandi, E., \& Zuluaga, G. P. (2014). Género, agroecología y soberanía alimentaria. Icaria: Barcelona, España, Spain, 240.

FAO. 2019. Sistema alimentario en México. Oportunidades para el campo mexicano en la Agenda 2030 de Desarrollo sostenible. Ciudad de México. 68pp. 